\title{
Cost-effectiveness of robotic hysterectomy versus abdominal hysterectomy in early endometrial cancer
}

Evelyn Lundin, Per Carlsson, Ninnie Borendal Wodlin, Lena Nilsson and Preben Kjölhede

The self-archived postprint version of this journal article is available at Linköping University Institutional Repository (DiVA):

http://urn.kb.se/resolve?urn=urn:nbn:se:liu:diva-171806

N.B.: When citing this work, cite the original publication.

Lundin, E., Carlsson, P., Borendal Wodlin, N., Nilsson, L., Kjölhede, P., (2020), Cost-effectiveness of robotic hysterectomy versus abdominal hysterectomy in early endometrial cancer, International Journal of Gynecological Cancer, 30(11), 1719-1725. https://doi.org/10.1136/ijgc-2020-001611

Original publication available at:

https://doi.org/10.1136/ijgc-2020-001611

Copyright: BMJ Publishing Group

http://group.bmj.com/ 
Original Research Article

\section{Cost-effectiveness of robotic hysterectomy versus abdominal hysterectomy in early endometrial cancer.}

by

Evelyn Serreyn Lundin ${ }^{1}, \mathrm{MD}$, Per Carlsson ${ }^{2}$, PhD, Ninnie Borendal Wodlin ${ }^{1}, \mathrm{MD}, \mathrm{PhD}$, Lena Nilsson ${ }^{3}$, MD, PhD, Preben Kjølhede ${ }^{1}$, MD, PhD

\section{$\underline{\text { Affiliations }}$}

${ }^{1}$ Department of Obstetrics and Gynecology in Linköping, and Department of Biomedical and Clinical Sciences, Linköping University, Linköping, Sweden

${ }^{2}$ Division of Society and Health, Department of Health, Medicine and Caring Sciences, Linköping University, Linköping, Sweden

${ }^{3}$ Department of Anesthesiology and Intensive Care in Linköping, and Department of Biomedical and Clinical Sciences, Linköping University, Linköping, Sweden

Corresponding author:

Evelyn Serreyn Lundin, MD

Department of Obstetrics and Gynecology,

University Hospital

58185 Linköping

Sweden

Phone +46 101030000

E-mail: Evelyn.Lundin@regionostergotland.se

\section{Funding}

The study was supported financially by grants from the Medical Research Council of South East Sweden, Linköping University and Region Östergötland.

\section{Conflict of interest statement}

None of the authors has any conflict of interest to declare. 


\section{Abstract}

Objectives: To compare total costs for hospital stay and postoperative recovery between robotic and abdominal hysterectomy in the treatment of early-stage endometrial cancer provided in an enhanced recovery after surgery (ERAS) setting. Costs were evaluated in relation to health impact, taking a societal perspective.

Methods: Cost analysis was based on data from an open randomized controlled trial in an enhanced recovery after surgery setting at a Swedish tertiary referral university hospital: 50 women with low-risk endometrial cancer scheduled for surgery between February 2012 and May 2016 were included; 25 women were allocated to robotic and 25 to abdominal hysterectomy. We compared the total time in the operating theater, procedure costs, postoperative care, and length of hospital stay, readmissions, informal care and sick leave as well as the health-related quality of life until six weeks after surgery. The comparison was made by using the EuroQoL group form with five dimensions and three levels (EQ-5D). The primary outcome measure was total cost, secondary outcomes were quality-adjusted life years (QALY) and cost per QALY.

Results: The robotic group recovered significantly faster as measured by the EQ-5D health index and gained 0.018 QALY until six weeks after surgery. Total costs were $20 \%$ higher for the robotic procedure (SEK 71,634 versus SEK 59,319). The total cost per QALY gained for women in the robotic group was slightly under SEK 700,000.

Conclusion: Robotic hysterectomy used in an enhanced recovery after surgery setting in the treatment of early endometrial cancer improved health within six weeks after the operation at a high cost for the health gained compared with abdominal hysterectomy. The productivity loss and informal care were lower for robotic hysterectomy, while health care had a higher procedure cost that could not be offset by the higher cost due to complications in the abdominal group. 
Keywords: cost-utility; endometrial cancer; health-related quality of life; quality-adjusted life year; robotic hysterectomy.

\section{Precis}

Although robotic hysterectomy in low-risk endometrial cancer provided a faster recovery measured by the health-related quality of life and QALY gained, the procedure was $20 \%$ more expensive than open surgery.

\section{Highlights}

- Robotic hysterectomy for low-risk endometrial cancer gained more QALY than open until six weeks after surgery.

- Robotic hysterectomy was more expensive than open, mainly explained by the higher costs for the robotic equipment.

- The total cost to gain one QALY was high in a societal perspective. 


\section{Introduction}

Laparoscopic surgery for early endometrial cancer has been advocated not only because of reduced bleeding, fewer postoperative complications and shorter hospital stay but also because of an overall better quality of life during recovery compared to abdominal surgery (1). However, the quality of the open technique has also improved over the years. Evidence exists that some of these proposed advantages of the minimally invasive surgery can be equalized, especially when the surgery is conducted in an enhanced recovery after surgery setting $(2,3)$.

The introduction of the robotic system for gynecological procedures, considered to overcome many of the challenges of conventional laparoscopy, was not evidence-based and the benefits of the technique are still being debated in relation to the high costs of the equipment $(4,5)$. Recent systematic review articles on robotic surgery reveal a lack of cost-effectiveness analyses in gynecologic oncology, especially with quality-adjusted life-year (QALY) as an outcome measure $(6,7)$.

We have previously published results from an open label, randomized controlled trial comparing robotic and abdominal hysterectomy treating women with low-risk endometrial cancer in an enhanced recovery after surgery model and showed a faster recovery for the robotic group (8). The present study describes a secondary outcome from that trial, evaluating health economics and QALY, The aim of this health-economic analysis was to determine whether the total costs and effects differed between the treatment groups in the study. The cost difference was then evaluated in relation to differences in health in order to calculate the incremental cost-effectiveness ratio (ICER) for the procedure. 


\section{Material and methods}

The study is based on an open label randomized controlled trial comparing robotic and abdominal hysterectomy, and was undertaken at the Department of Obstetrics and Gynecology at the University Hospital in Linköping, Sweden. Women admitted for treatment of FIGO stage I, low-risk endometrial cancer (endometrioid adenocarcinoma, FIGO grades 1 and 2) and scheduled for hysterectomy and bilateral salpingo-oophorectomy with peritoneal washings between February 2012 and May 2016 were eligible for the study. The inclusion and exclusion criteria, randomization process, perioperative care, postoperative follow-up and study flow chart have been described previously (8). The Regional Ethical Review Board in Linköping approved the trial (Dnr 2011/108-31), and the study was registered in ClinicalTrial.gov (NCT 01526655).

EuroQol group 5-dimension 3-levels questionnaire (EQ-5D)

A commonly used validated generic questionnaire, the EQ-5D (9) assessed the health-related quality of life. A unique EQ-5D health state is defined by combining one of three levels of severity from each of the five dimensions. This health state can be converted into utility, using a weighted health state index by applying scores from EQ-5D value sets elicited from the general population, to calculate the health-related quality of life. The index ranges from zero to one. Zero indicates the state of death and one indicates full health. The EQ-5D has been validated under Swedish circumstances (10). The form was completed preoperatively, during the first eight days postoperatively, and then once weekly until the six-week postoperative visit.

Assessment of QALY and ICER

QALY is a generic measure of disease burden, including both the quality and the quantity of life lived. A year of life lived in perfect health is worth one QALY and death is assigned a value of 0 QALYs. The change in utility value induced by the treatment is multiplied by the 
duration of the treatment effect to provide the number of QALYs gained. In order to estimate the QALY gained, we used the difference in EQ-5D health state index between the robotic and the abdominal group. For the first eight days, the measured EQ-5D index was obtained for each day. For the second to the sixth weeks postoperatively the EQ-5D index was obtained on days $14,21,28,35$ and 42 . The missing EQ-5D index on the intermediate days in these weeks was replaced by the average of the index value on the day of measurement and the value the week before.

ICER is used to summarize the cost-effectiveness of a health care intervention compared to an alternative intervention and is calculated by dividing the cost differences between the compared interventions by the difference in QALY for each group, respectively. It represents the average incremental cost associated with one additional unit of the measure of effect, in this context QALY.

\section{Direct costs}

Relevant direct costs related to hospital stay and follow-up six weeks postoperatively were calculated using costs from the year 2018. The costs were calculated in Swedish Crowns (SEK). The average exchange rate in 2018 determined by the Swedish National Bank was US $\$ 100=$ SEK 869.21 and for $€ 100=$ SEK 1,025.67. The estimation of costs comprised relevant resources related to the operation, such as staff and procedure-specific material costs, and costs due to unplanned visits and readmissions after discharge from the ward. Even sick leave and informal care were considered.

The hospital internal fees, a fixed "once-and-for-all cost" in three categories for the operation, based on the calculated average real cost of the surgery depending on the extent of the intervention, and the "per minute charge" for the surgery, a variable cost depending on the duration of surgery, were used, obtained from the cost accounting records from the University Hospital, Linköping. The variable cost for total time in the operating theater exclusive time of 
surgery included mean yearly salaries from the year 2018 for the nurse anesthetist, the operating theater nurse and the assistant nurse working at that time (11). The salary for the anesthesiologist was not included here because the procedure of spinal analgesia with intrathecal morphine (25 minutes) and induction of general anesthesia (15 min) and intermittent supervision of the anesthetic course was considered equal in each group and thereby only proportionately increased costs in the groups. The cost for the nurse anesthetist, operating theater nurse and assistant nurse was calculated as a mean of the yearly salary in Sweden multiplied by 1.43 to include the social fees regulated by law (12). A full-time employment covers approximately 200 days annually and the time spent in the operating theater is approximately eight hours per day. This yields a cost per minute of SEK 7.1 for the nurse anesthetist, SEK 7.2 for the operating theater nurse and SEK 5.1 for the assistant nurse.

The procedure cost for the robotic hysterectomy covers the purchase price of the robot, the da Vinci ${ }^{\circledR}$ Si System with camera and optics (SEK 14 022,750 in 2007) with a depreciation time of eight years, and the yearly maintenance fee (SEK 1307,600 in 2007). The first year of maintenance was free of charge. For the present annual caseload of 300 robotic operations, the procedure price tag was SEK 9,657 per robotic operation compared with a possible optimal annual caseload of 500 operations, and with two operations a day over 50 weeks, the price was SEK 5,794 per procedure. Surgical equipment like the instrument containers, one for the surgical robotic instruments (Maryland bipolar, needle driver and monopolar scissors) and one for the other accessory robotic instruments was specified, taking into account the lifetime of the instruments limited to ten separate procedures and the lifetime of the container of ten years. The costs for sterilization of instruments, draping and extra material used in the operation were also calculated. The procedure cost for the abdominal hysterectomy consisted of one laparotomy container with reusable surgical instruments including the disposable- bipolar vessel sealing device (Ligasure ${ }^{\circledR}$, Medtronic) required in 
order to perform the surgery according to the technique applied in minimal invasive surgery. We assumed the lifetime of the abdominal hysterectomy instruments and instrument container to be 10 years and calculated the price for an annual caseload of 300 and 500 hysterectomies.

The length of hospital stay in the gynecological ward was determined as time in hours from arrival at the operation theater to discharge from the ward. Costs for hospital stay in the ward were derived from cost accounting records and amounted to SEK 9,240 per 24 hours in 2018. This cost comprised salaries for all types of personnel involved, laboratory analyses, the use of pharmaceuticals, core facilities, heating and cleaning.

The number of unplanned visits and readmissions after discharge from the hospital was registered in both groups. The readmission cost was equal to the hospital ward cost, SEK 385 per hour. The unit price of SEK 3,005 was assigned for an outpatient visit to a physician and SEK 2,196 for a visit to a nurse.

\section{Indirect costs}

Duration of sick leave was defined as all days from the day of surgery to the day of return to work to the same extent as the women had preoperatively. The women received sick leave as long as they considered it necessary. Costs for this productivity loss were estimated by the human capital approach, based on the average annual income for women in Sweden 2018, aged 20-64 years, which was SEK 559,416 including social benefits $(11,12)$ giving a cost of productivity loss per day of SEK 1533.

The weekly reported informal care after discharge, given by a relative, friend or neighbor, was summarized in hours for all weeks. Based on the human capital approach, the informal care was valued by its opportunity cost based on a study by Johannesson et al. (13), with one hour of leisure time valued as $35 \%$ of the gross wage rate. According to the general income in Sweden for the working population in 2018 of SEK 34,600 per month, considering 
social fees $(11,12)$ the informal care costs were valued at approximately SEK 71 per hour. The costs in SEK for all items are summarized in Table 1.

\section{Statistics}

Sample size estimation was based on the primary outcome measure of quality of life, the EQ5D health index. With an $\alpha=0.05$ and $1-\beta=0.90$, the sample size was estimated to be 50 women including a drop-out of $10 \%$, based on the assumption that the EQ-5D health index differed by at least 0.2 units between the two groups, and given the standard deviation of the EQ-5D index (0.2) obtained from previously published data from our department (14). All analyses were performed according to intention- to-treat principles. Data were expressed as median and interquartile range or number and percentage. Univariate analyses were performed with a student's T-test or Mann-Whitney U-test for continuous data and a Chisquare test or Fisher's exact test for nominal data. All tests were two-sided, and the significance level was set at $p<0.05$. The software TIBCO Statistica ${ }^{\mathrm{TM}}$, version 13.5 (TIBCO Software Inc, Palo Alto, CA 94303, USA) was used to carry out the statistical analyses. 


\section{Results}

The participant characteristics and resource utilization are demonstrated in Table 2. The data showed no statistically significant differences between the groups except for a shorter time in the operating theater, and also shorter operation and anesthesia time for the abdominal group. Although two thirds of the women were retired and thus did not need sick leave, no significant differences concerning number of days on sick leave or hours of informal care provided were seen between the study groups. The number of readmissions to the hospital or unplanned visits did not show statistically significant differences. However, the number of unplanned visits was numerically higher in the abdominal group.

Table 3 demonstrates the proportional total costs for the robotic and abdominal hysterectomy groups respectively, divided into direct and indirect costs. The robotic hysterectomy cost SEK 12,315 more per patient than the abdominal hysterectomy. This was mainly attributed to higher direct costs for the robotic procedure whereas the indirect costs were substantially lower for the robotic hysterectomy compared with the abdominal hysterectomy. However, by increasing the annual number of procedures from 300 to 500 the total costs for the robotic surgery would decrease by $30 \%$ to SEK 8,456 per patient.

The results of the EQ-5D health state index are represented in Figure 1. The completeness of filling in the EQ-5D forms was $98.54 \%$.

The average quality-of- life weights for both groups were calculated as the sum of the EQ-5D index in the group for the 43 days divided by 43 days $(37.59 / 43=0.87$ for the robotic and $31.12 / 43=0.72$ for the abdominal group). Each woman in the robotic group gained 0.15 . To obtain the QALY gained the average quality-of-life weights were multiplied by 0.12 (43 days/365 days per year) and resulted in 0.018 QALYs compared with the abdominal group.

The ICER showed a cost for one QALY gained in the robotic group of 12,315/0.018= SEK 684,167 based on 300 and SEK 469,778 based on 500 operations a year. 


\section{Discussion}

This study showed that women undergoing robotic hysterectomy in an enhanced recovery after surgery program for treatment of early endometrial cancer gained better health than those undergoing abdominal hysterectomy, though there was a high cost for the improvement.

Robotic hysterectomy in low-risk endometrial cancer was $20 \%$ more expensive than abdominal, mainly due to the procedure costs comprising both the acquisition and maintenance of the $d a$ Vinci ${ }^{\circledR}$ robot and the robotic instrument costs. This is also confirmed by other studies (5-7). In 2017, Iavazzo et al. (6) published a review article about cost-benefit analysis in gynecological oncology emphasizing the absence of competition on the market as one of the most important factors causing these high robotic costs.

The occurrence of complications and readmissions after open surgery for endometrial cancer is an important post-discharge cost factor that the robotic procedure could reduce as emphasized by Leitao, et al. (15) and Lau, et al. (16). In our study the high robotic costs could only partially be offset by the lower cost for readmissions and unplanned outpatient visits in the abdominal group. Maybe the extent of the open surgery, both concerning the skin incision and the intra-abdominal surgery, plays a role. Saheli et al. (17) found lower total health costs after robotic surgery in a randomized trial of high-risk endometrial cancer comparing robotic and abdominal hysterectomy including pelvic and para-aortic lymphadenectomy. The lower costs were mainly due to a shorter hospitalization. However, that trial have important shortcomings concerning evaluation of the health care costs. Indirect costs and even some direct costs were not reported. Besides, the trial was not conducted in a standardized ERAS framework making the comparison of the two surgical modes uncertain.

In contrast to Herling, et al. (18) we failed to demonstrate that robotic hysterectomy was cheaper than abdominal hysterectomy concerning total costs. Nor could we demonstrate the shorter length of hospital stay that was shown in other trials $(4,5)$. It is likely that our results 
reflect the strict use of an enhanced recovery after surgery program treating both groups similarly. Traditional care regimes are often conservative, favoring the expected improved outcome after robotic surgery, with a longer length of hospital stay after open surgery when an enhanced recovery after surgery program is not applied. With standardized discharge criteria, the average length of hospital stay could be reduced to 36 hours in both groups, illustrating that many other factors affect the length of hospital stay. Economically a shorter length of stay is a prerequisite that compensates for the higher procedure costs of the robot, as argued by many authors. A recently published large Danish study (19) evaluated the longterm resource consequences of introducing robotic surgery in women undergoing robotic, conventional laparoscopic or open surgery for early-stage endometrial cancer. They found no significant difference in days of hospitalization when controlling for time trends. The robotic surgery generated additional costs, driven by the primary surgery, and no long-term cost savings were seen.

The total time in the operating theater, including surgery and anesthesia time did not turn out to be decisive for the incremental costs due to the robotic procedure even when this parameter differed significantly between the groups in our study. This was also as shown by Pellegrino et al. (20) in benign hysterectomy.

Sick leave plays a key role in indirect costs. In prostate cancer the costs for sick leave following open surgery counterbalance the increased costs for the robotic procedure (21). Unfortunately, the study was not performed in an enhanced recovery after surgery model. In our study the women operated with the abdominal technique needed on average six days more sick leave, although not equalizing the robotic costs.

Informal care is a less visible part of total care in terms of societal costs. More hours were spent with patients in the abdominal hysterectomy group, but at a low cost in both groups. 
The gain in QALY in the robotic group was low for the individual woman and the estimated cost per QALY was higher than accepted in Sweden for new interventions, taking the severity of the condition into consideration (22).

The strengths of the trial are the randomized design and the use of an enhanced recovery after surgery program, offering the women similar perioperative care to optimize recovery $(23,24)$. Our study is the first trial within gynecologic oncology surgery with a cost-utility analysis (25). The small sample size could be seen as a limitation. However, the sample size was powered for the primary outcome, the EQ-5D health index (8) that is crucial for the health-economic evaluation. The use of hospital accounting records and salaries from the year 2018 implies that the total cost evaluation is not exact but proportional which validates the cost comparison. Another limitation may be the limited time period of follow-up used in the calculation of QALY. We believe that the period is justified by the fact that the EQ-5D index after six weeks had recovered to a level better than the preoperative level and the risk for costs associated with the surgery were low after six weeks.

\section{Conclusion}

Robotic hysterectomy for low-risk endometrial cancer results in a faster recovery in quality of life at a higher total cost than abdominal hysterectomy, mainly explained by the higher costs for the robot and the equipment. More effort should be made to prevent complications following abdominal hysterectomy in order to accept this procedure as a treatment option for endometrial cancer patients.

\section{Acknowledgment}

We especially want to thank the research nurses Åsa Rydmark Kersley, Linda Shosholli and Gunilla Gagnö for their dedicated work with the patients and on the project. We are also 
indebted to nurse specialist in surgery Jeanette Tjerngren and to the chief economist Anders Östberg for providing the pricelist for procedure and hospital costs.

\section{Disclosure of interests}

None of the authors has any conflicts of interest to declare.

\section{Contribution to authorship}

The study was planned by PK, LN and NBW and conducted by ESL, PK, LN and NBW. Data were analyzed by ESL, PK, PC and LN. ESL is the main author of the draft. All authors ESL, PK, PC, LN and NBW contributed to the interpretation of the results, the elaboration of the manuscript and approval of the final version.

\section{Funding}

The study was supported financially by grants from the Medical Research Council of South East Sweden, Linköping University and the Region Östergötland. 


\section{References}

1. He H, Zend D, Ou H, et al. Laparoscopic treatment of endometrial cancer: systematic review. J Minim Invasive Gynecol 2013;20(4):413-23.

2. Nelson G, Altman AD, Nick A, et al. Guidelines for pre- and intra-operative care in gynecologic/oncology surgery: Enhanced Recovery After Surgery (ERAS®) Society recommendations--Part I. Gynecol Oncol. 2016;140(2):313-22.

3. Nelson G, Altman AD, Nick A, et al. Guidelines for postoperative care in gynecologic/oncology surgery: Enhanced Recovery After Surgery (ERAS $®)$ Society recommendations--Part II. Gynecol Oncol. 2016;140(2):323-32.

4. Park DA, Lee DH, Kim SW, et al. Comparitive safety and effectiveness of robotassisted laparoscopic hysterectomy versus conventional laparoscopy and laparotomy for endometrial cancer: A systematic review and meta-analysis. Eur J Surg Oncol. 2016; 42 (9):1303-14.

5. Kristensen SE, Mosgaard BJ, Rosendahl M, et al. Robot-assisted surgery in gynecological oncology: current status and controversies on patient benefits, cost and surgeon conditions - a systematic review. Acta Obstet Gynecol Scand. 2017;96(3):27485.

6. Iavazzo C, Gkegkes ID. Cost-benefit analysis of robotic surgery in gynaecological oncology. Best Pract Res Clin Obstet Gynaecol. 2017;45:7-18.

7. Tandogdu Z, Vale L, Fraser C, et al. A systematic review of economic evaluations of the use of robotic assisted laparoscopy in surgery compared with open or laparoscopic surgery. Appl Health Econ Health Policy. 2015;13(5):457-67.

8. Lundin ES, Wodlin NB, Nilsson L, et al. A prospective randomized assesment of quality of life between open and robotic hysterectomy in early endometrial cancer. Int $J$ Gynecol Cancer 2019; March 28, doi: 10.1136/ijgc-2019-000285. 
9. EuroQol Group. EuroQol--a new facility for the measurement of health-related quality of life. Health Policy. 1990;16(3):199-208.

10. Burström K, Johannesson M, Diderichsen F. Swedish population health-related quality of life results using the EQ-5D. Qual Life Res 2001;10: 621-35.

11. Statistiska Centralbyrån (Statistics Sweden). Folkmängd efter region, civilstånd, ålder och kön. År 1968-2018. [Internet], Stockholm 2019 [cited 2019 October 7] (in Swedish) Available from:

http://www.statistikdatabasen.scb.se/pxweb/sv/ssd/START_BE_BE0101_BE0101A /BefolkningNy/?rxid=f45f90b6-7345-4877-ba25-9b43e6c6e299

12. Hjalte F, Olofsson S, Persson U. Sjukdomsbördan vid migrän i Sverige - en enkätstudie av resurskonsumtion och livskvalitet. [in Swedish] IHE Rapport 2018:4, IHE: Lund.

13. Johannesson M, Borgquist L, Jonsson B, et al. The costs of treating hypertension- an analysis of different cut-off points. Health Policy. 1991;18(2):141-50.

14. Wodlin NB, Nilsson L, Kjølhede P. Health-related quality of life and postoperative recovery in fast-track hysterectomy. Acta Obstet Gynecol Scand. 2011;90(4):362-8.

15. Leitao MM Jr, Bartashnik A, Wagner I, et al. Cost-effectiveness analysis of robotically assisted laparoscopy for newly diagnosed uterine cancers. Obstet Gynecol. 2 2014;123(5):1031-7.

16. Lau S, Vaknin Z, Ramana-Kumar AV, et al. Outcomes and cost comparisons after introducing a robotics program for endometrial cancer surgery. Obstet Gynecol. 2012;119(4):717-24.

17. Salehi S, Åvall-Lundqvist E, Legerstam B et al. Robot-assisted laparoscopy versys laparotomy for infrarenal paraaortic lymphadenectomy in women with high-risk endometrial cancer: A randomised controlled trial. Eur J Cancer. 2017;79:81-9. 
18. Herling SF, Palle C, Møller AM, et al. Cost-analysis of robotic-assisted laparoscopic hysterectomy versus total abdominal hysterectomy for women with endometrial cancer and atypical complex hyperplasia. Acta Obstet Gynecol Scand. 2016;95(3):299-308.

19. Korsholm M, Gyrd-Hansen D, Mogensen O, et al. Long term resource consequences of a nationwide introduction of robotic surgery for women with early stage endometrial cancer. Gynecol Oncol. 2019;154 (2):411-9.

20. Pellegrino A, Damiani GR, Fachechi G, et al. Cost analysis of minimally invasive hysterectomy vs open approach performed by a single surgeon in an Italian center. $J$ Robot surg. 2017;11(2):115-21.

21. Forsmark A, Gehrman J, Angenete E, et al. Health Economic Analysis of Open and Robot-assisted Laparoscopic Surgery for Prostate Cancer Within the Prospective Multicentre LAPPRO Trial. Eur Urol. 2018;74:816-24.

22. Neumann A, Lindholm L, Norberg M, et al. The cost-effectiveness of interventions targeting lifestyle change for the prevention of diabetes in a Swedish primary care and community based prevention program. Eur J Health Econ. 2017;18:905-19.

23. Iavazzo C, Gkegkes ID. Enhanced recovery programme in robotic hysterectomy. Br J Nurs. 2015;24(16):S4-8.

24. Kehlet H, Wilmore DW. Evidence-based surgical care and the evolution of fast-track surgery. Ann Surg. 2008;248(2):189-98.

25. Korsholm M, Sorensen J, Mogensen O, et al. A systematic review about costing methodology in robotic surgery: evidence for low quality in most of the studies. Health Econ Rev. 2018;8:21. 
Cost-effectiveness of robotic hysterectomy

\section{Figure Legends}

Figure 1. EQ-5D weighted health state index in relation to occasion of measurement. 
Table 1. Mean cost (in SEK) per item and operation with a yearly caseload of 300 and 500 operations

\begin{tabular}{|c|c|c|c|c|}
\hline & \multicolumn{4}{|c|}{ Costs per item (SEK) } \\
\hline & \multicolumn{2}{|c|}{ Robotic hysterectomy } & \multicolumn{2}{|c|}{ Abdominal hysterectomy } \\
\hline & \multicolumn{2}{|c|}{ Annual case load } & \multicolumn{2}{|c|}{ Annual case load } \\
\hline & $\begin{array}{c}300 \\
\text { operations }\end{array}$ & $\begin{array}{c}500 \\
\text { operations }\end{array}$ & $\begin{array}{c}300 \\
\text { operations }\end{array}$ & $\begin{array}{c}500 \\
\text { operations }\end{array}$ \\
\hline Fixed cost for the operation (preparation and closing of theatre, basic equipment) & 5,818 & 5,818 & 5,818 & 5,818 \\
\hline Costs for two surgeons (per minute) & 46 & 46 & 46 & 46 \\
\hline Variable cost for staff in operation room exclusive surgeons for time of surgery (per minute) & 93 & 93 & 93 & 93 \\
\hline Variable cost for nursing staff in operation room exclusive time of surgery (per minute) & 19 & 19 & 19 & 19 \\
\hline Robotic equipment (including camera and optics; purchase and maintenance) & 9,657 & 5,794 & - & - \\
\hline Surgical instruments and two instrument container for robotic surgery & 7,927 & 7,924 & - & - \\
\hline Surgical instruments and instrument container for laparotomy (including disposable Ligasure®) & - & - & 2,018 & 2,011 \\
\hline Extra equipment and material for the surgery & 4,000 & 4,000 & 2,315 & 2,315 \\
\hline Draping for surgery & 632 & 632 & 562 & 562 \\
\hline Sterilization robotic and surgical instruments & 2,521 & 2,521 & 615 & 615 \\
\hline Fixed cost for time in post anesthesia care unit & 2,399 & 2,399 & 2,399 & 2,399 \\
\hline Hospital care in the gynecological ward (per hour) & 385 & 385 & 385 & 385 \\
\hline Costs readmission for 24 hours & 9,240 & 9,240 & 9,240 & 9,240 \\
\hline Outpatient visit to physician (per visit) & 3,005 & 3,005 & 3,005 & 3,005 \\
\hline Outpatient visit to nurse (per visit) & 2,196 & 2,196 & 2,196 & 2,196 \\
\hline Costs for sick leave (per day) & 1,533 & 1,533 & 1,533 & 1,533 \\
\hline Costs for informal care (per hour) & 71 & 71 & 71 & 71 \\
\hline
\end{tabular}

Exchange rate per December 31, 2018: US \$ $100=$ SEK 869.21; $€ 100=$ SEK 1,025.67 
Table 2. Participant characteristics and resource utilization

\begin{tabular}{lll}
\hline & \multicolumn{1}{c}{$\begin{array}{c}\text { Robotic } \\
\text { hysterectomy } \\
(\mathrm{n}=25)\end{array}$} & $\begin{array}{c}\text { Abdominal } \\
\text { hysterectomy } \\
(\mathrm{n}=24)\end{array}$ \\
\hline Age (years) & $68(63-72)$ & $67(59-75)$ \\
Body mass index (kg/m²) & $28.2(24.9-32.6)$ & $28.3(24.6-30.8)$ \\
Parity & $2.0(2.0-2.0)$ & $2.0(1.5-3.0)$ \\
ASA classification $\quad$ Class I (no. of women) & $9(36 \%)$ & $11(46 \%)$ \\
$\quad$ Class II (no. of women) & $15(60 \%)$ & $12(50 \%)$ \\
$\quad$ Class III (no. of women) & $1(4 \%)$ & $1(4 \%)$ \\
Employed (no. of women) & $8(32 \%)$ & $9(37 \%)$ \\
Time of surgery (minutes)\# & $70(60-90)$ & $56(49-84)$ \\
Estimated per-operative blood loss (mL) & $50(25-50)$ & $50(25-100)$ \\
Time of anesthesia (minutes)\# & $147(130-170)$ & $115(92-122)$ \\
Time in operation room (minutes)\# & $176(165-200)$ & $145(129-156)$ \\
Time in PACU (hours) & $4.4(4.0-5.1)$ & $5.2(3.9-5.8)$ \\
Length of hospital stay, de facto (hours) & $53(51-54)$ & $51(50-52)$ \\
Length of hospital stay, discharge criteria were met (hours) & $36(36-36)$ & $36(36-54)$ \\
Sick leave (no. of women) & $8(32 \%)$ & $9(37 \%)$ \\
Sick leave (days) & $25(17-30)$ & $31(26-35)$ \\
Receiving informal care (no. of women) & $12(48 \%)$ & $10(42 \%)$ \\
Informal care (hours) & $5.5(2.5-22.5)$ & $16.8(7-34)$ \\
Readmissions to hospital (no. of patients) & $0(0 \%)$ & $3(12.5 \%)$ \\
Unplanned visits to physician (no. of visits/ no. of patients) & $3 / 1(4 \%)$ & $12 / 7(29.2 \%)$ \\
Unplanned visits to nurse (no. of visits/ no. of patients) & $3 / 2(8 \%)$ & $14 / 6(25 \%)$ \\
\hline Fign
\end{tabular}

Figures denote median (and interquartile range) or number (and percent). ASA, American Society of Anesthesiologists risk of classification; PACU, Post Anesthesia Care Unit. \#Time of surgery $(p=0.048)$, anesthesia time $(p<0.0001)$ and time in operation room $(p<0.0001)$ differed significantly between the groups in the univariate analysis. 
Table 3. Total mean cost per patient (in SEK) based on the study population of 25 robotic (RH) and 24 abdominal hysterectomies (AH) extrapolated to an annual caseload of 300 and 500.

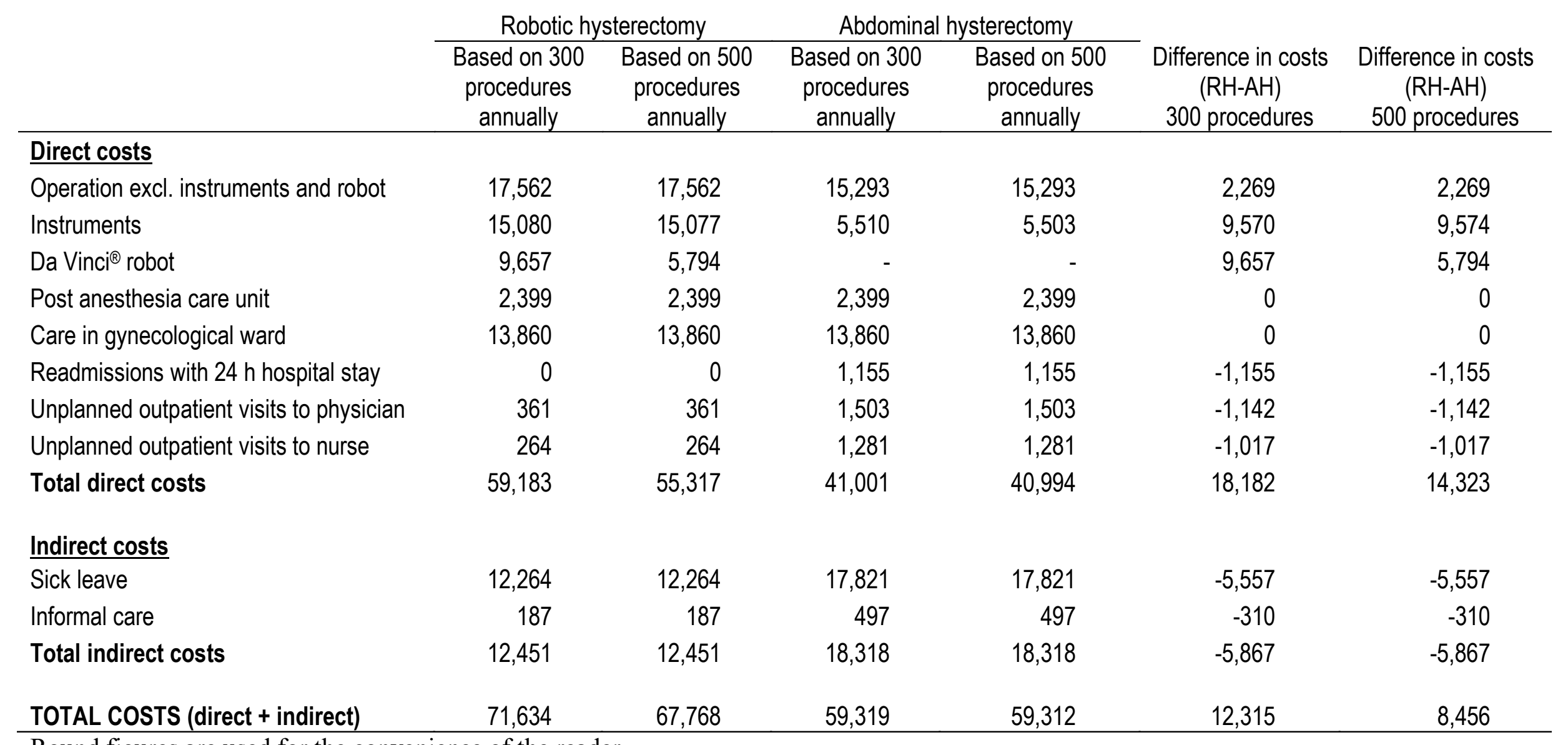

Round figures are used for the convenience of the reader.

Exchange rate per December 31, 2018: US \$100= SEK 869.21; € $100=$ SEK 1,025.67 


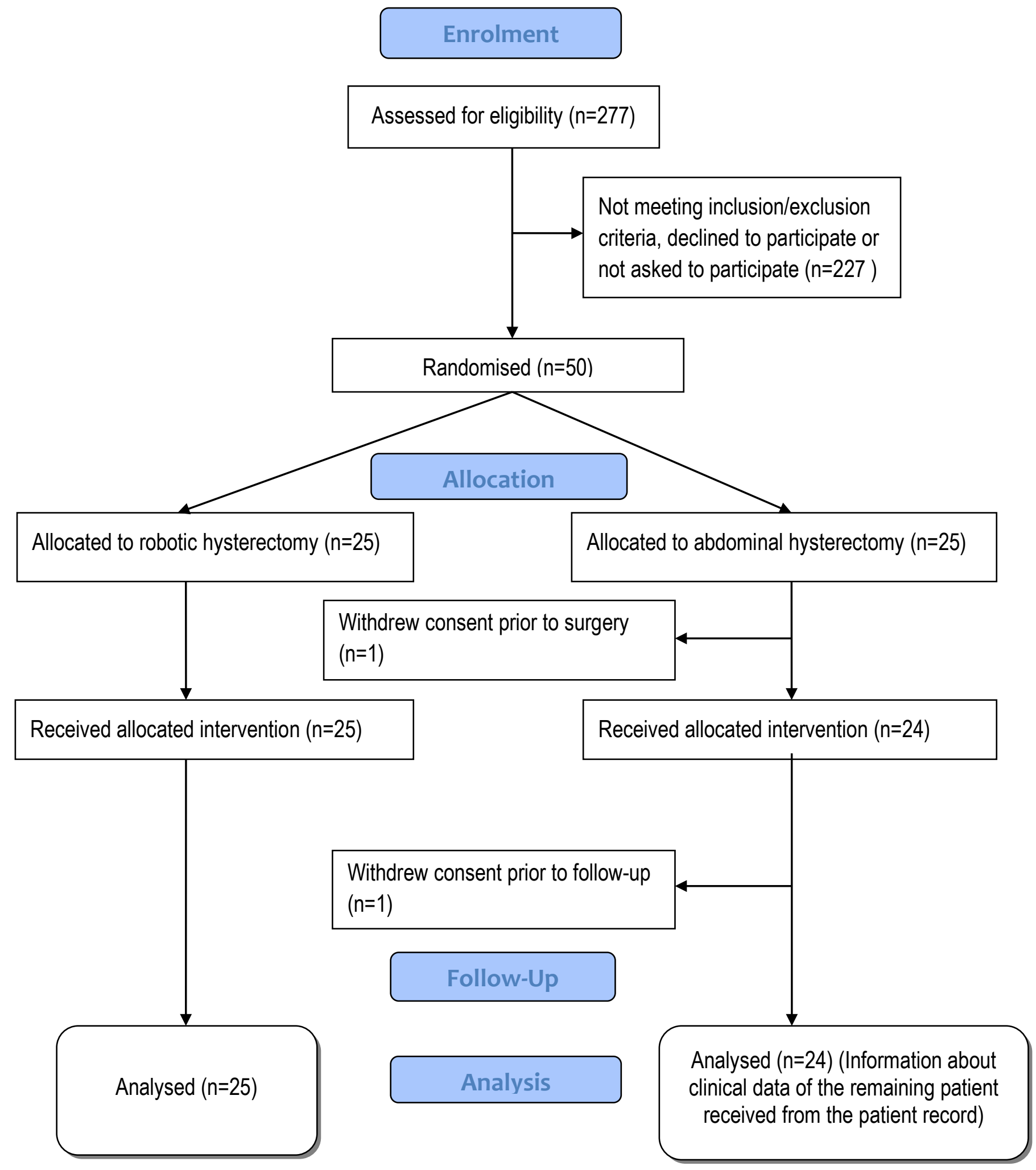

Figure 1. Flow chart of participants in the study. 


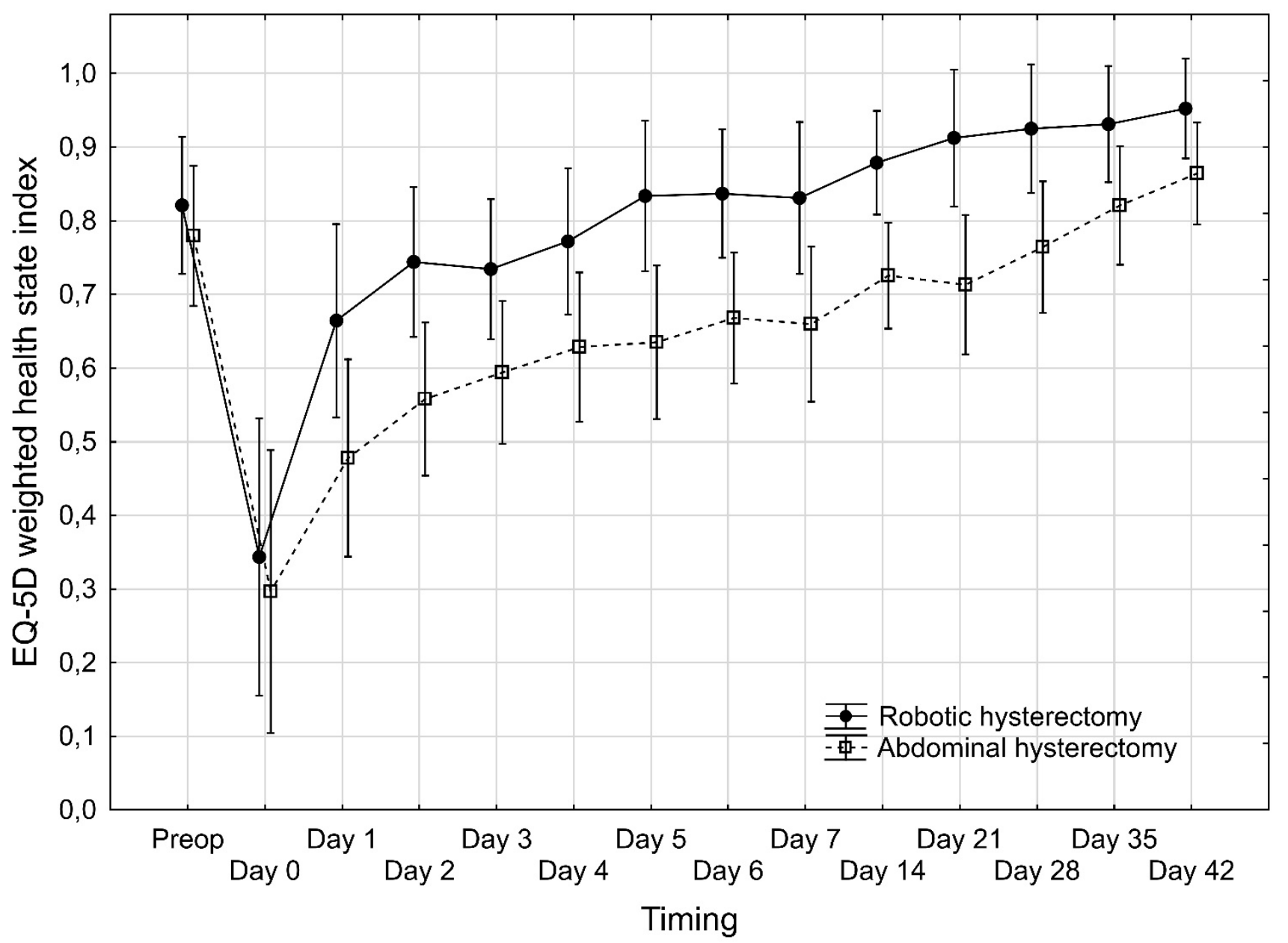

Figure 2. EQ-5D weighted health state index in relation to occasion of measurement. Plots represent means and bars represent $95 \%$ confidence intervals. No significant difference was observed between the two groups at baseline preoperatively (Mann-Whitney U-test). 